\section{OPEN ACCESS}

Edited by: Wafik S. El-Deiry,

Brown University, United States

Reviewed by:

Pamela Bond Cassidy,

Oregon Health and Science University,

United States

Nathan Dolloff,

Medical University of South Carolina

United States

*Correspondence: Ning Deng

tdengn@jnu.edu.cn Jianfu Zhao

zhaojianfu@jnu.edu.cn

${ }^{\dagger}$ These authors have contributed equally to this work

Specialty section: This article was submitted to

Cancer Molecular Targets and Therapeutics,

a section of the journal

Frontiers in Oncology

Received: 20 July 2020 Accepted: 04 January 2021 Published: 25 February 2021

Citation:

Zhang S, Huang J, Zhang L, Gu J,

Song $Q$, Cai $Y$, Zhong J, Zhong $H$, Deng $Y$, Zhu W, Zhao J and Deng N (2021) Fermentation, Purification, and

Tumor Inhibition of a DisulfideStabilized Diabody Against Fibroblast Growth Factor-2.

Front. Oncol. 11:585457. doi: 10.3389/fonc.2021.585457

\title{
Fermentation, Purification, and Tumor Inhibition of a Disulfide- Stabilized Diabody Against Fibroblast Growth Factor-2
}

\author{
Simin Zhang $^{1 \dagger}$, Jiahui Huang ${ }^{1 \dagger}$, Ligang Zhang ${ }^{1 \dagger}$, Jiangtao Gu ${ }^{1}$, Qifang Song ${ }^{1}$, \\ Yaxiong Cai ${ }^{1}$, Jiangchuan Zhong ${ }^{1}$, Hui Zhong ${ }^{2}$, Yanrui Deng ${ }^{1}$, Wenhui Zhu ${ }^{1}$, \\ Jianfu Zhao $^{3 *}$ and Ning Deng ${ }^{1 *}$
}

1 Guangdong Province Engineering Research Center for Antibody Drug and Immunoassay, Department of Biology, Jinan University, Guangzhou, China, 2 Biomedicine Translational Institute, Jinan University, Guangzhou, China, ${ }^{3}$ Cancer Diagnosis and Therapy Research Center, Department of Oncology of the First Affiliated Hospital, Jinan University, Guangzhou, China

Angiogenesis is considered one of the hallmarks of cancer and plays a critical role in the development of tumor. Fibroblast growth factor 2 (FGF-2) is a member of the FGF family and participates in excessive cancer cell proliferation and tumor angiogenesis. Thus, targeting FGF-2 was considered to be a promising anti-tumor strategy. A disulfidestabilized diabody (ds-Diabody) against FGF-2 was produced in Pichia pastoris (GS115) by fermentation and the anti-tumor activity was analyzed. The novel 10-L fed batch fermentation with newly designed media was established, and the maximum production of the ds-Diabody against FGF-2 reached 210.4 mg/L. The ds-Diabody against FGF-2 was purified by $\mathrm{Ni}^{2+}$ affinity chromatography and DEAE anion exchange chromatography. The recombinant ds-Diabody against FGF-2 could effectively inhibit proliferation, migration, and invasion of melanoma and glioma tumor cells stimulated by FGF-2. Furthermore, xenograft tumor model assays showed that the ds-Diabody against FGF2 had potent antitumor activity in nude mice by inhibiting tumor growth and angiogenesis. The tumor growth inhibition rate of melanoma and glioma was about 70 and 45\%, respectively. The tumor angiogenesis inhibition rate of melanoma and glioma was about 64 and $51 \%$, respectively. The results revealed that the recombinant ds-Diabody against FGF-2 may be a promising anti-tumor drug for cancer therapy.

Keywords: fibroblast growth factor 2, angiogenesis, disulfide-stabilized diabody, melanoma cancer, glioma cancer

\section{INTRODUCTION}

Malignant tumor has become the second leading cause of death worldwide, and there were about 9.6 million cancer-related deaths in the year before last (1). Angiogenesis is considered one of the hallmarks of cancer and plays a critical role in the development of tumor (2). These new blood vessels mainly transport nutrients, oxygen, and metabolic wastes for the growth and metastasis of tumors (3). Therefore, anti-angiogenesis is a very important strategy for cancer therapy designed to disrupt the vascular supply (4). 
Fibroblast growth factor 2 (FGF-2) is a member of the FGF family that functions in regulating both normal and abnormal angiogenesis $(5,6)$. It is upregulated in inflammatory stimuli and in tumors (7-9). It has been considered that the active FGF-2 could mediate the formation of new blood vessels (10). FGF-2 integrates the extracellular domain of fibroblast growth factor receptor (FGFR) and heparan sulfate proteoglycans (HSPG) to induce the autophosphorylation of FGFR intracellular tyrosine kinase domain and lead to the activation of complex signal transduction pathways $(11,12)$. They participate in the development of tumors as oncogenes by inducing mitogenic and survival signals, promoting tumor cell invasion and metastasis, promoting epithelial-mesenchymal transition, promoting angiogenesis, and participating in tumor recurrence and drug resistance (13). Thus, targeting FGF-2 for inhibition of tumor growth and angiogenesis was considered to be a promising therapeutic strategy.

In the last decade, FGF/FGFR inhibitors were new focuses in the field of cancer therapy. The tyrosine kinase inhibitors (TKIs), such as TKI258, cediranib, and brivanib, can block receptor signaling through competitive inhibition of ATP binding with the cytoplasmic domain of EGFR, FGFR, and VEGFR, but they are less capable of achieving efficient inhibitions and also increasing side effects $(14,15)$. The other attractive option as a selective FGF/ FGFR inhibitor is the monoclonal antibody (mAb). mAbs offer some significant advantages over TKIs, including specificity for targeting to a particular receptor. This circumvents certain toxicity issues and enhances the anti-tumor response (16). A disulfidestabilized diabody (ds-Diabody) against FGF-2, a kind of small molecular monoclonal antibody, was composed of variable heavy chain (VH) and variable light chain (VL) with a short connecting linker (five amino acid residues) to induce formation of the scFv dimer, and its disulfide bonds were introduced to maintain stability and affinity. Small molecule antibodies get more and more attention for their good tissue penetration and low immunogenicity (17). They may have potential applications in tumor-targeted therapy.

In this study, we mainly reported the production of ds-Diabody against FGF-2 by high density fermentation in recombinant Pichia pastoris and its effects on tumor angiogenesis and tumor growth in vitro and in vivo.

\section{MATERIALS AND METHODS}

\section{Cell Culture}

Human melanoma cancer cells (A375) (ATCC, Manassas, VA, USA) were cultured in RPMI-1640 medium (Gibco, Grand Island, NY, USA), and the human glioma cancer cells (U87) (ATCC, Manassas, VA, USA) were cultured in DMEM medium (Gibco) supplemented with $10 \%$ fetal bovine serum (FBS, Gibco) and $1 \%$ penicillin/ streptomycin (Gibco) in an incubator at $37^{\circ} \mathrm{C}$ with $5 \% \mathrm{CO} 2$.

\section{Animals}

Female BALB/c nude mice (6-7 weeks old) were purchased from Beijing Sibeifu Biotechnology Co., Ltd. (China). Animals were acclimatized to the facilities for 10 days. The mice were housed in specific pathogen-free environment, and the treatment of animals at $22 \pm 2^{\circ} \mathrm{C}$ and $50 \pm 10 \%$ relative humidity with water and food (laboratory rodent chow, Shanghai, China) allowed ad libitum. The treatment of animals was approved by the Institutional Animals Care and Use Committee on animal research in Jinan University, Guangzhou, China.

\section{The Production of ds-Diabody Against FGF-2 by Fermentation}

The recombinant Pichia pastoris GS115-pPICZ $\alpha$ A-ds-Diabody constructed in our previous study (17) was inoculated into $300 \mathrm{ml}$ of YPG medium (per liter contained tryptone, $20.0 \mathrm{~g}$; Yeast extract, $10.0 \mathrm{~g}$; glycerol, $10.0 \mathrm{~g}$ ) and cultivated at $28^{\circ} \mathrm{C}$ with $250 \mathrm{rpm}$ shaking for $36 \mathrm{~h}$. The medium was transferred to $3 \mathrm{~L}$ of BSM medium (per liter contained histidine, $13.3 \mathrm{~g}$; glycerol, $40.0 \mathrm{~g} ; \mathrm{K}_{2} \mathrm{SO}_{4}, 18.0 \mathrm{~g} ; \mathrm{MgSO}_{4} \cdot 7 \mathrm{H}_{2} \mathrm{O}, 14.9 \mathrm{~g} ; \mathrm{KOH}, 4.13 \mathrm{~g} ; \mathrm{CaSO}_{4}$, $0.9 \mathrm{~g} ; \mathrm{H}_{3} \mathrm{PO}_{4}, 27.0 \mathrm{ml} ; \mathrm{H}_{2} \mathrm{SO}_{4}, 5.0 \mathrm{ml}$ and $4.0 \mathrm{ml}$ of PTM trace salts which was per liter consisted of $\mathrm{CuSO}_{4} \cdot 5 \mathrm{H}_{2} \mathrm{O}, 6.0 \mathrm{~g}$; KI, $0.09 \mathrm{~g} ; \mathrm{MnSO}_{4} \cdot \mathrm{H}_{2} \mathrm{O}, 3.0 \mathrm{~g} ; \mathrm{H}_{3} \mathrm{BO}_{3}, 0.02 ; \mathrm{MoNa}_{2} \mathrm{O}_{4} \cdot 2 \mathrm{H}_{2} \mathrm{O}, 0.2 \mathrm{~g}$; $\mathrm{CoCl}_{2}, 0.5 \mathrm{~g} ; \mathrm{ZnCl}_{2}, 20.0 \mathrm{~g} ; \mathrm{FeSO}_{4} \cdot 7 \mathrm{H}_{2} \mathrm{O}, 65.0 \mathrm{~g}$ and biotin, $0.2 \mathrm{~g}$ ) in a $10-\mathrm{L}$ standard mechanical agitated fermenter bioreactor (T\&J Bio-engineering Co., Ltd, Shanghai, China). At the stage of fed-batch cultivation (phase I), the culture was maintained at $28^{\circ} \mathrm{C}$, pH 5.0 and $20 \%$ dissolved oxygen (DO). After $18-24 \mathrm{~h}$, glycerol was exhausted, and the DO increased to $100 \%$ rapidly. The fed-batch phase (phase II) was initiated by feeding limited glycerol to allow further cell growth. 50\% (v/v) glycerol supplemented with PTM trace salts $(12 \mathrm{ml} / \mathrm{L})$ was added at a rate of $12 \mathrm{ml} / \mathrm{h} / \mathrm{L}$. The methanol fed-batch (strategy I) or the sorbitol/methanol fed-batch (strategy II) was taken in phase III after the cell wet weight reached 180-220 g/L. For strategy I, methanol was added with PTM trace salts $(12 \mathrm{ml} / \mathrm{L})$ at the speed of $1 \mathrm{ml} / \mathrm{h} / \mathrm{L}$, and the feeding speed of methanol increased by a ratio of $20 \%$ per hour until it reached $6 \mathrm{ml} / \mathrm{h} / \mathrm{L}$. For strategy II, the sorbitol was co-fed with methanol at a fixed feeding ratio of 1:4 (g/g). In phase III, the condition of fermentation was adjusted to $28^{\circ} \mathrm{C}, \mathrm{pH} 6.0$ and the $\mathrm{DO} \geq 20 \%$. The cell wet weight was measured, and the supernatant was collected by centrifuging the culture at $12,000 \mathrm{~g}$ for $15 \mathrm{~min}$ at $4^{\circ} \mathrm{C}$. The expression of dsDiabody was analyzed by reduced SDS-PAGE.

\section{Purification of ds-Diabody Against FGF-2}

The supernatant was precipitated with ammonium sulfate and dialyzed against a binding buffer. It was purified by $\mathrm{Ni}^{2+}$-affinity chromatography due to His-tag at C-terminal of recombinant protein and by DEAE weak-anion exchange chromatography for higher purity according to the manufacture protocol. The eluted fractions were dialyzed with phosphate buffered saline (PBS) using $10-\mathrm{kDa}$ cut-off membrane ultrafiltration devices (GE healthcare) and stored at $-20^{\circ} \mathrm{C}$ for further analysis.

\section{SDS-PAGE Electrophoresis Analysis}

The molecular mass of ds-Diabody was determined by reduced sodium dodecyl sulfate polyacrylamide gel electrophoresis (SDSPAGE). Each sample was mixed with $5 \times$ loading buffer (Sangon, 
Shanghai, China) and heated for $10 \mathrm{~min}$ at $100^{\circ} \mathrm{C}$. Samples were then separated under reducing condition on $12 \%(\mathrm{~m} / \mathrm{v})$ trisglycine gels (Sangon) using a Mini-gel system (Bio-Rad). The proteins in the gel were stained with Coomassie (Sangon) and analyzed using the Automatic Analysis System of Electrophoresis Gel Imaging (Tanon Science Technology Co. Ltd, Shanghai, China).

\section{IDENTIFICATION OF DS-DIABODY AGAINST FGF-2 BY WESTERN BLOT}

After SDS-PAGE, the proteins were transferred onto polyvinylidenedifluoride (PEDV) membrane (Millipore, Bedford, USA). The membrane was block with $5 \%(\mathrm{~m} / \mathrm{v})$ nonfat milk dissolved in phosphate buffered saline supplemented with $0.05 \%(\mathrm{v} / \mathrm{v})$ Tween-20 (PBS-T) at $37^{\circ} \mathrm{C}$ for $2 \mathrm{~h}$ and probed with anti-His tag antibody (1:1,000 dilution) (362601, Biolegend, San Diego, CA) at $4^{\circ} \mathrm{C}$ overnight, followed by incubation with the horseradish peroxidase (HRP) conjugated goat anti-mouse IgG (1:8,000 dilution) $\left(405306\right.$, Biolegend) at $37^{\circ} \mathrm{C}$ for $30 \mathrm{~min}$. The membrane was washed three times for $3 \mathrm{~min}$ with PBS-T between incubation steps. Immunoreactive bands were visualized by Immobilon Western Chemiluminescent HRP Substrate (Millipore, Bedford, USA) according to protocol and were performed with Automatic Gel Image Analysis System (Tanon Science Technology Co. Ltd).

\section{Antigen Binding Activity of ds-Diabody Against FGF-2 by ELISA}

The 96-well plates were coated with FGF-2 (50 ng/well) (\#P09038, $\mathrm{R} \& \mathrm{D}$, Minneapolis, $\mathrm{MN}, \mathrm{USA})$ at $4^{\circ} \mathrm{C}$ overnight, and the BSA (50 ng/well) (A3311, Sigma) coated wells were control group for nonspecific protein binding. The next day, the plates were washed and blocked with $5 \%$ non-fat milk at $37^{\circ} \mathrm{C}$ for $2 \mathrm{~h}$. The purified dsDiabody was quantified by BCA Protein Assay Kit (Thermo Scientific, Rockford, IL, USA) according to the protocol and was serially diluted $(15.63,7.81,3.91,1.95,0.98,0.49,0.24,0.12,0.06$, 0.03 , and $0.02 \mu \mathrm{g} / \mathrm{ml}$ ) with PBS buffer and incubated at $37^{\circ} \mathrm{C}$ for $1.5 \mathrm{~h}$. The anti-c-Myc antibody (1:2,000 dilution) (sc-40, Santa Cruz, Cambridge, UK) was incubated at $37^{\circ} \mathrm{C}$ for $1.5 \mathrm{~h}$ binding to cMyc tag of ds-Diabody. The HRP-conjugated goat anti-mouse IgG (1:4,000 dilution) (405306, Biolegend, San Diego, CA) was added and incubated at $37^{\circ} \mathrm{C}$ for $30 \mathrm{~min}$. The plates were washed three times for 3 min with PBS-T between incubation steps. The reaction wells were developed with $\mathrm{TMB}\left(3,3^{\prime}, 5,5^{\prime}\right.$-tetramethylbenzidine) (Sigma, St. Louis, MO, USA), and the reaction was stopped with sulfuric acid. The absorbance values of each well were measured at $450 \mathrm{~nm}$ using the ELISA reader (BioTek, Highland Park, Winooski, VT, USA).

\section{Cell Proliferation Assays}

The human melanoma cancer cells (A375) (2,000 cells/well) and the human glioma cancer cells (U87) (1,000 cells/well) were seeded in 96 -well plates and incubated overnight at $37^{\circ} \mathrm{C}$ in a $5 \%$ $\mathrm{CO}_{2}$ incubator. The cells were then serum-starved cultured in the medium with $0.5 \%$ FBS for $12 \mathrm{~h}$ and treated with serially diluted ds-Diabodies (6.25-100 $\mu \mathrm{g} / \mathrm{ml})$ with $15 \mathrm{ng} / \mathrm{ml} \mathrm{FGF-2} \mathrm{for} 48 \mathrm{~h}$. The full-length human IgG against FGF-2 $(6.25-100 \mu \mathrm{g} / \mathrm{ml})$ was the positive control, and control IgG $(6.25-100 \mu \mathrm{g} / \mathrm{ml})$ was negative control. The proliferation of tumor cells was assayed using Cell Counting Kit-8 (CCK-8) (Dojindo Laboratories, Kumamoto, Japan) according to the manufacturer's protocol. The proliferation inhibition rate was calculated according to the $\mathrm{OD}_{450}$ values measured in an ELISA reader (BioTek).

\section{Migration Inhibition of ds-Diabody on Human Melanoma and Glioma Cells}

The effects of the ds-Diabody against FGF-2 on migration of A375 cells and U87 cells were evaluated by wound healing assays. A375 cells $\left(2.5 \times 10^{5}\right.$ cells/well $)$ and U87 cells $\left(2.0 \times 10^{5}\right.$ cells/ well) were seeded in 6 -well plates and incubated at $37^{\circ} \mathrm{C}$ for $12-$ $24 \mathrm{~h}$ in a $5 \% \mathrm{CO}_{2}$ incubator. When cells grew to confluence, a cell-free area was introduced using a pipette tip. After that, cells were washed with PBS, cultured in the medium with $0.5 \%$ FBS and treated with ds-Diabody $(100 \mu \mathrm{g} / \mathrm{mL})$ with $15 \mathrm{ng} / \mathrm{mL}$ FGF-2. The full-length human IgG against FGF-2 $(100 \mu \mathrm{g} / \mathrm{ml})$ was positive control, control IgG $(100 \mu \mathrm{g} / \mathrm{ml})$ was negative control, and PBS was vehicle control, respectively. Cells were captured at 0,12 , and $24 \mathrm{~h}$ and the migration rate was calculated.

\section{Invasion Assays}

The effects of the ds-Diabody against FGF-2 on invasion of A375 cells and U87 cells were evaluated by invasion assays. The transwell chamber with $8 \mu \mathrm{m}$ filter (BD Biosciences, San Jose, CA, USA) was coated with $100 \mu \mathrm{l}$ Matrigel matrix (BD Biosciences) which was diluted (1:30) with serum-free medium. After serum-starved for $12 \mathrm{~h}, \mathrm{~A} 375$ cells $\left(2.0 \times 10^{4}\right.$ cells/well $)$ and the U87 cells $\left(1.0 \times 10^{4}\right.$ cells/well) were transferred to the transwell chambers and cultured in serum-free medium with $15 \mathrm{ng} / \mathrm{ml} \mathrm{FGF-2} \mathrm{and} \mathrm{treated} \mathrm{with} \mathrm{ds-}$ Diabody $(100 \mu \mathrm{g} / \mathrm{ml})$ for $16 \mathrm{~h}$. $600 \mu \mathrm{l}$ medium with 10\% FBS was added in the lower chambers as a chemoattractant. After the treatment, cells on the lower side of the filter were fixed with $70 \%$ ethanol, stained by $0.1 \%$ crystal violet (Meryer, Shanghai, China) and imaged with a computerized imaging system. Full-length human IgG against FGF-2 $(100 \mu \mathrm{g} / \mathrm{ml})$, control IgG $(100 \mu \mathrm{g} / \mathrm{ml})$, and PBS were positive control, negative control, and vehicle control, respectively.

\section{Western Blot Assays of Phosphorylation of Akt and MAPK}

A375 cells $\left(2.0 \times 10^{5}\right.$ cells/well $)$ and U87 cells $\left(1.0 \times 10^{5}\right.$ cells/ well) were seeded in 6 -well plates and incubated at $37^{\circ} \mathrm{C}$ overnight in a $5 \% \mathrm{CO}_{2}$ incubator. Cells were serum-starved cultured in the medium with $0.5 \%$ FBS for $12 \mathrm{~h}$. The medium was exchanged containing $15 \mathrm{ng} / \mathrm{ml} \mathrm{FGF-2,} \mathrm{0.5 \%} \mathrm{FBS,} \mathrm{and} \mathrm{cells}$ were treated with serially diluted ds-Diabody $(0,50,100$, and 200 $\mu \mathrm{g} / \mathrm{ml}$ ) at $37^{\circ} \mathrm{C}$ for $30 \mathrm{~min}$. Cells were then washed with PBS and lysed with RIPA lysis buffer (Beyotime Biotechnology, Suzhou, China) at $4^{\circ} \mathrm{C}$ for $10 \mathrm{~min}$. The lysates were centrifuged at 12,000 $\mathrm{g}$ for $10 \mathrm{~min}$ at $4^{\circ} \mathrm{C}$. The total proteins in supernatant were quantified by using Pierce BCA Protein Assay Kit (Thermo 
Scientific). Proteins were separated by reduced SDS-PAGE and transferred to PVDF membrane (Millipore). The membrane was blocked with $5 \%$ non-fat milk at $37^{\circ} \mathrm{C}$ for $2 \mathrm{~h}$ and incubated with rabbit anti-t/p-MAPK (Cell Signaling Technology, Danvers, MA, USA) and rabbit anti-t/p-Akt (Cell Signaling Technology) at $4^{\circ} \mathrm{C}$ overnight. The membrane was then incubated with the HRPconjugated goat anti-rabbit IgG (Cell Signaling Technology) for $30 \mathrm{~min}$ at $37^{\circ} \mathrm{C}$. The blots were detected with the Immobilon Western Chemiluminescent HRP Substrate (Millipore) according to manufacturer's protocol. The rabbit anti-GAPDH antibody (Cell Signaling Technology) was used as reference control.

\section{Tumor Xenograft Models}

For melanoma and glioma tumor xenograft experiments, BALB/cnu mice $(n=7)$ were randomly assigned into three treatment groups (seven mice per group). A375 cells $\left(2.0 \times 10^{6}\right.$ cells in $\left.100 \mu \mathrm{l}\right)$ and U87 cells $\left(5.0 \times 10^{6}\right.$ cells in $\left.100 \mu \mathrm{l}\right)$ were s.c. injected into the right shoulder of female $\mathrm{BALB} / \mathrm{c}$ nude mice. When tumors were palpable, mice were i.v. injected with the ds-Diabody $(5 \mathrm{mg} / \mathrm{kg}$ in $100 \mu \mathrm{l}$ PBS $)$ six times at 3 days intervals. Avastin antibody $(2 \mathrm{mg} / \mathrm{kg}$ in $100 \mu \mathrm{l}$ PBS $)$ and PBS $(100 \mu \mathrm{l})$ were positive control and vehicle control, respectively. Body weights and tumor sizes were measured every 3 days. Tumor volumes $\left(\mathrm{mm}^{3}\right)$ were calculated according to the formula $\mathrm{V}=0.52 \mathrm{ab}^{2}$ ( $\mathrm{a}=$ length of tumor; $\mathrm{b}=$ width of tumor). At the end of 15 days, nude mice were euthanized, and tumors were stripped for weighing to calculate tumor growth inhibition rate and for immunohistochemistry assays. Tumor growth inhibition rates were calculated as ( 1 - the average tumor weight of treated groups/ the average tumor weight of PBS group) $\times 100 \%$.

\section{Immunohistochemistry Assays for CD31}

The immunohistochemistry assays for CD31 were performed to determine the intratumoral microvessel density. CD31 was detected in immersion fixed paraffin sections of tumor tissues using antiCD31 rabbit polyclonal antibodies (ab28364; Abcam, Cambridge, $\mathrm{UK}$ ) at $10 \mu \mathrm{g} / \mathrm{ml}$ overnight at $4^{\circ} \mathrm{C}$ followed by incubation with goat anti-rabbit IgG H\&L HRP Polymer Antibody (ab205718; Abcam, Cambridge, UK). Tissues were stained using DAB (brown) and counterstained with hematoxylin (blue).

\section{Statistical Analysis}

Data were expressed as the mean and standard deviation (SD). P $<0.05$ was considered statistically significant. Assays were performed at least three times independently. Statistical comparisons between two groups were analyzed by one-way ANOVA with the least significant difference test (LSD test).

\section{RESULTS}

\section{The Production of ds-Diabody Against FGF-2 by Fermentation}

The recombinant Pichia pastoris GS115-pPICZ $\alpha$ A-ds-Diabody was constructed in our previous study (Figure 1A) (17). The maximum production ds-Diabody was $47.4 \mathrm{mg} / \mathrm{L}$ and wet cell weight (WCW) was $393.1 \mathrm{~g} / \mathrm{L}$ in methanol fed-batch (strategy I)
(Figure S1). the Pichia pastoris expression system is impressive and easy to handle, and the process optimizations are required to achieve maximum production of the target proteins especially in induction phase. Among the co-feeding strategies, co-carbon source (sorbitol) feeding strategy is as important as methanol feeding strategy (18). Thus, in order to further improve the production of the target protein, sorbitol/methanol fed-batch (strategy II) was used in our study. Surprisingly, the maximum ds-Diabody concentration reached $210.4 \mathrm{mg} / \mathrm{L}$ and WCW was $390.0 \mathrm{~g} / \mathrm{L}$ in sorbitol/methanol fed-batch phase (Figures 1B). The production of ds-Diabody was increased by 4.4 times when mixed feeding of sorbitol and methanol. The ds-Diabody expressed successfully and was observed a band of about $35 \mathrm{kDa}$ using reduced SDS-PAGE (Figure 1D).

\section{Purification of ds-Diabody Against FGF-2}

The supernatant was precipitated with ammonium sulfate and dialyzed against a binding buffer. Supernatant was then purified through $\mathrm{Ni}^{2+}$-affinity chromatography due to His-tag at C-terminal of recombinant protein and through anion-exchange chromatography for higher purity. The purified recombinant protein was observed as a single band of about $35 \mathrm{kDa}$ using reduced SDS-PAGE and western blot analysis with $>95 \%$ purity (Figure 1E).

\section{Antigen Binding Activity of the ds-Diabody Against FGF-2 Using Indirect ELISA Assays}

To further determine the binding activity and specificity of dsDiabody, the indirect ELISA assays were performed. When the concentration of the ds-Diabody was $0.49 \mu \mathrm{g} / \mathrm{ml}$, the value of $\mathrm{OD}_{450}$ in FGF-2 group was 1.08, while 0.12 in BAS group. The results demonstrated that ds-Diabody bound specifically to FGF2 and had a high binding ability (Figure 1C).

\section{Proliferation Inhibition of ds-Diabody on Human Melanoma and Glioma Cells}

A375 cells and U87 cells were shown to express FGF-2 at high levels (19-21). Additionally, FGF-2 strongly stimulates the proliferation and migration of tumor cells (5). To investigate bioactivity of the ds-Diabody to inhibit FGF-2-induced proliferation of in vitro, CCK-8 assays were performed. The results showed that dsDiabody displayed dose-dependent inhibitory activity on A375 cells and U87 cells. When the concentration of the ds-Diabody was $6.25,12.5,25.0,50.0$ and $100.0 \mu \mathrm{g} / \mathrm{ml}$, the A375 cells proliferation inhibition rate was about $16,21,30,32$, and $37 \%$, respectively (Figure 2A); the U87 cells' proliferation inhibition rate was about 19, 20, 24, 25, and 29\%, respectively (Figure 2B), which showed similar efficacy to full-length human IgG (positive control). Control IgG showed no inhibitory effect.

\section{Migration Inhibition of ds-Diabody on Tumor Cells}

Wound healing assays were used to reveal the effects of ds-Diabody on migration of A375 cells and U87 cells. A375 cells $\left(2.5 \times 10^{5}\right.$ cells/ well) and U87 cells $\left(2.0 \times 10^{5}\right.$ cells/well $)$ were seeded in 6 -well plates and incubated at $37^{\circ} \mathrm{C}$ in a $5 \% \mathrm{CO} 2$ incubator. When cells grew to 
A

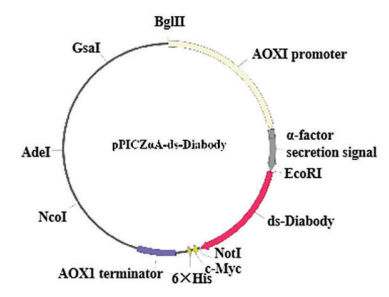

B

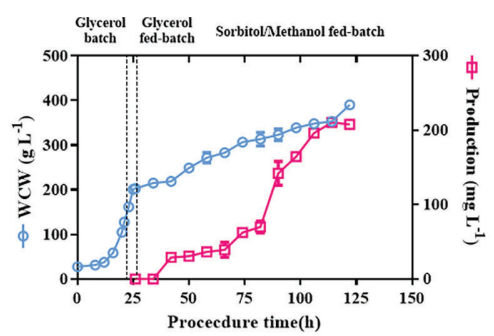

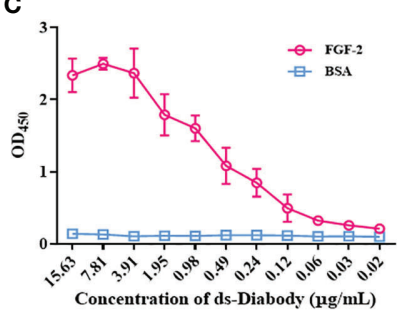
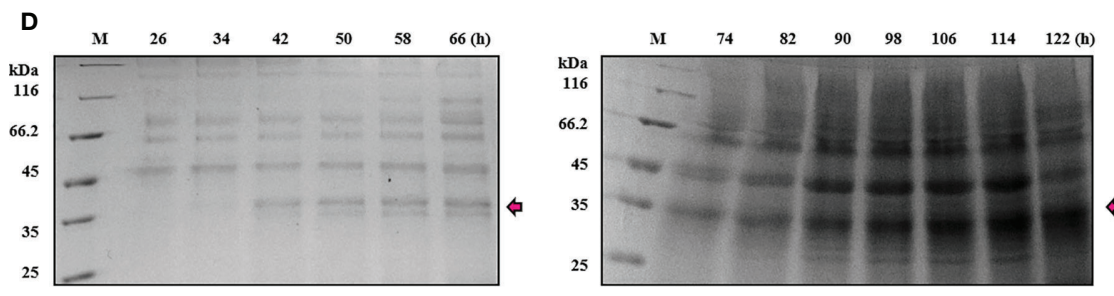

E

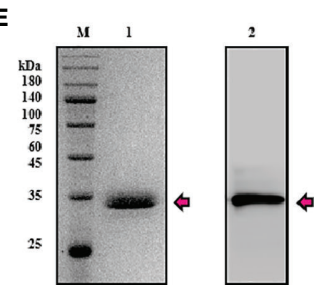

FIGURE 1 | The fermentation, purification and binding activity studies of ds-Diabody against FGF-2 in Pichia pastoris. (A) Schematic illustration of the recombinant expression plasmid of pPICZ $\alpha$ A-ds-Diabody. (B) The curve of cell wet weight and the production of ds-Diabody against FGF-2 in Pichia pastoris fermentation for strategy II. (C) The antigen combined activity of ds-Diabody against FGF-2 by ELISA. (D) The SDS-PAGE assays of ds-Diabody against FGF-2 in fermentation for strategy II. (E) The purification of ds-Diabody. Lane M, marker; Lane 1, the SDS-PAGE assay of the purified ds-Diabody in reducing condition; Lane 2: the Western blot assays of the purified ds-Diabody.

A

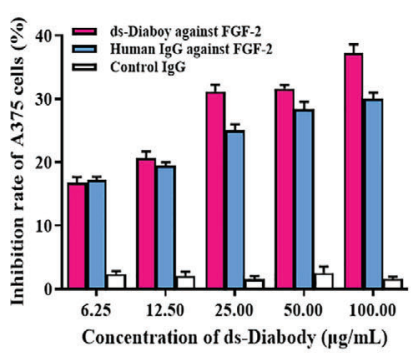

$\mathbf{E}$

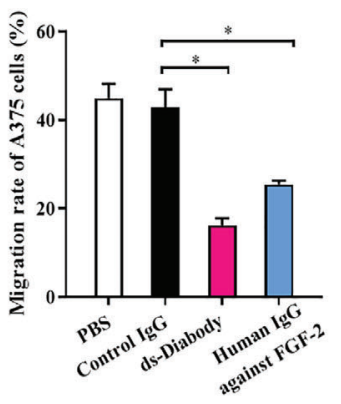

B

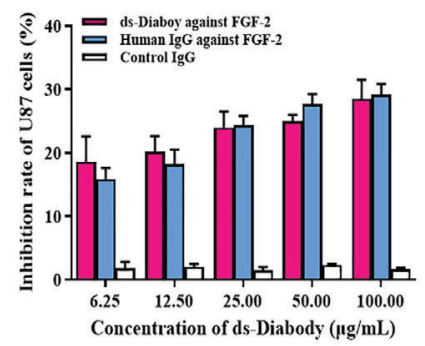

$\mathbf{F}$

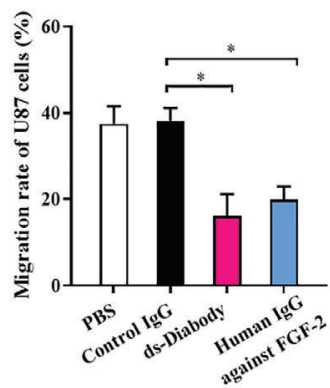

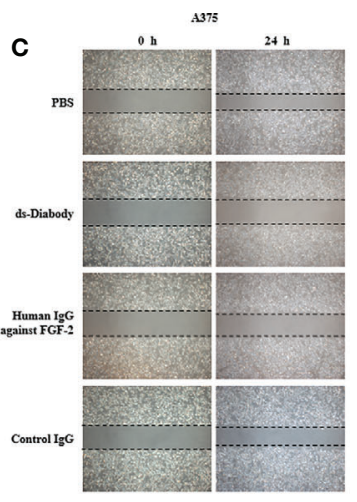

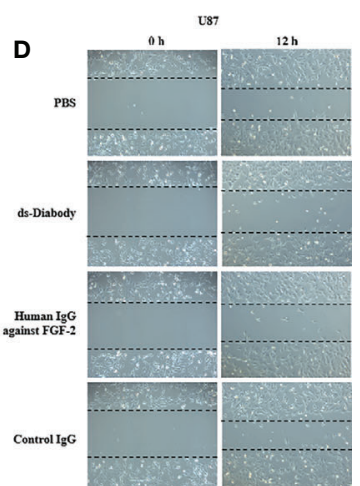

FIGURE 2 | The tumor proliferation and migration inhibition of ds-Diabody against FGF-2. (A) The proliferation inhibition of ds-Diabody on A375 cells.

(B) The proliferation inhibition of ds-Diabody on U87 cells. (C) The migration of A375 cells in different groups. (D) The migration of U87 cells in different groups.

(E) The quantitative analysis of the migration rate of A375 cells in different groups. (F) The quantitative analysis of the migration rate of U87 cells in different groups.

The results presented as the mean $\pm \mathrm{SD}$ in triplicate. ${ }^{*} \mathrm{p}<0.05$ versus Irrelevant Group. 
confluence, a cell-free area was introduced using a pipette tip, and cells were cultured in the medium with $0.5 \%$ FBS and treated with ds-Diabody $(100 \mu \mathrm{g} / \mathrm{ml})$ with $15 \mathrm{ng} / \mathrm{ml}$ FGF-2. The migration rate of A375 cells for $24 \mathrm{~h}$ in the group of the ds-Diabody, the full-length human IgG against FGF-2, the irrelevant IgG and PBS were 16, 25, 43 , and $45 \%$, respectively (Figures $2 \mathrm{C}, \mathbf{E}$ ). The migration rate of U87 cells for $12 \mathrm{~h}$ in groups of the ds-Diabody against FGF-2, the full-length human IgG against FGF-2, the control IgG and PBS were $16,20,38$, and $38 \%$, respectively (Figures 2D, F). It was shown that the migration of A375 and U87 cells were significantly inhibited in ds-Diabody-treated group compared to control.

\section{Invasion Inhibition of ds-Diabody on Tumor Cells}

The invasion assays were used to reveal the effects of the dsDiabody on invasion of A375 cells and U87 cells. After serumstarved for $12 \mathrm{~h}, \mathrm{~A} 375$ cells $\left(2.0 \times 10^{4}\right.$ cells/well $)$ and the U87 cells $\left(1.0 \times 10^{4}\right.$ cells/well $)$ were transferred to the transwell chambers. Cells cultured in upper chamber with serum-free medium and 15 $\mathrm{ng} / \mathrm{ml}$ FGF-2 were chemo-attracted to the lower chamber containing complete medium. The invasion rate of A375 cells in the group of the ds-Diabody, the full-length human IgG against FGF-2, the control IgG and PBS were 55, 77, 96, and $100 \%$, respectively (Figure 3A). The invasion rate of U87 cells in the group of the ds-Diabody, the full-length human IgG against FGF-2, the control IgG and PBS were 46, 75, 96, and $100 \%$, respectively (Figure 3B). It was shown that the ds-Diabody could significantly inhibit the invasion of A375 cells and U87 cells.

\section{Western Blot Assay of Phosphorylation of Akt and MAPK}

FGF-2 could promote tumor proliferation, migration, and angiogenesis as a key angiogenic factor (5). To further confirm whether ds-Diabody was capable of inhibiting FGF-2 signaling, a phosphorylation assay of Akt and MAPK was performed. The starved A375 cells and U87 cells were treated with different concentrations of ds-Diabody together with FGF-2. The cells were then lysed, and proteins in lysates were separated with reduced SDSPAGE and detected by western-blot. The results showed that the dsDiabody could effectively block the phosphorylation activation of Akt and MAPK in a dose-dependent manner (Figures 3C, D), which suggesting an effective inhibitory function of ds-Diabody
A

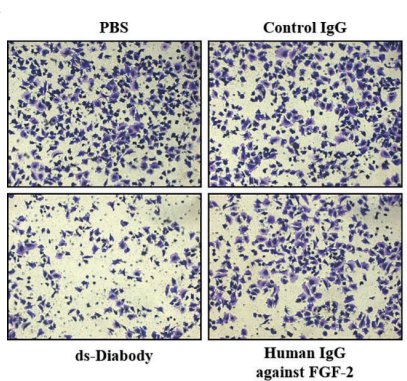

C

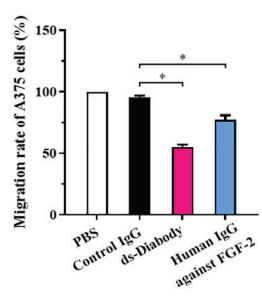

B

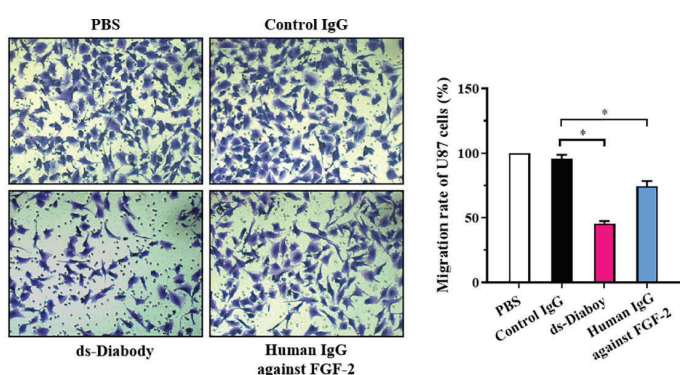

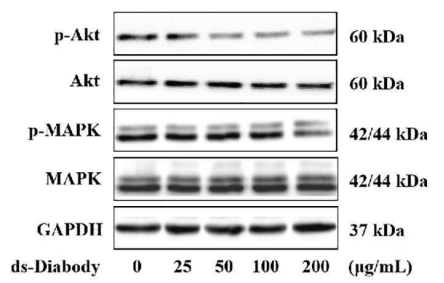
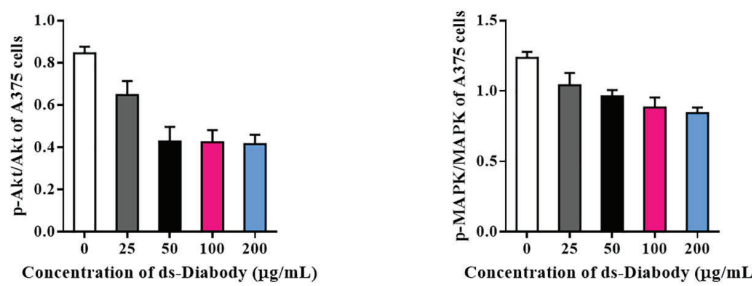

D
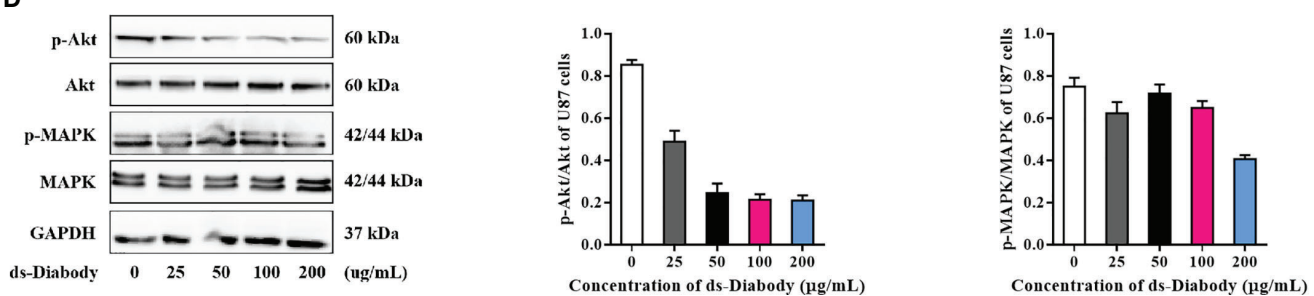

FIGURE 3 | The invasion inhibition and phosphorylation assays of Akt and MAPK in tumor cells treated with ds-Diabody against FGF-2. (A) The invasion and the quantitative analysis of A375 cells in different groups. (B) The invasion and the quantitative analysis of U87 cells in different groups. (C) Western blot assays of Akt and MAPK phosphorylation in A375 cells treated by ds-Diabody against FGF-2. (D) Western blot assays of Akt and MAPK phosphorylation of U87 cells treated by ds-Diabody against FGF-2. The results presented as the mean \pm SD in triplicate. ${ }^{*} p<0.05$ versus Irrelevant Group. 
against FGF-2-stimulated proliferation, migration and angiogenesis in A375 cells and U87 cells.

\section{The ds-Diabody Against FGF-2 Suppressed Tumors Growth and Angiogenesis in Xenograft Models}

The antitumor activity of ds-Diabody was evaluated in xenograft mouse models. The model was established via subcutaneously injection of A375 cells $\left(2.0 \times 10^{6}\right.$ cells $)$ or U87 cells $\left(5.0 \times 10^{6}\right.$ cells) on the shoulder of female $\mathrm{BALB} / \mathrm{c}$ nude mice. The result showed that the tumor volumes and tumor weights of the dsDiabody-treated group were significantly lower compared to the control group. The growth inhibition rate of A375 tumor in the dsDiabody group was about $70 \%$ and in the Avastin group was about $54 \%$ (Figures 4A-C). The growth inhibition rate of U87 tumor in the ds-Diabody group was about $45 \%$ and in the Avastin group was about 36\% (Figures 5A-C). The weights of BABL/c-nu mice treated with ds-Diabody were relatively stable (Figures 4D, 5D).
Moreover, immunohistochemical analysis showed that ds-Diabody could reduce the expression of CD31 in A375 (Figure 4E) and U87 (Figure 5E) tumor tissues. The angiogenesis inhibition rate of A375 tumor and U87 tumor treated by ds-Diabody was about 64 and $51 \%$, respectively, suggesting that ds-Diabody could suppress tumor growth by inhibiting tumor angiogenesis.

\section{DISCUSSION}

Currently, several expression systems are used to produce recombinant proteins such as bacteria, yeasts and mammals (22). Compare to bacteria expression systems, yeast expression systems like Pichia pastoris are preferred tools for high expression of properly folded secretory proteins and limited production of endogenous secretory proteins $(23,24)$. Moreover, they are easy to handle, less costly, and well suited for fermentation processes (25). Mammalian cell lines such as $\mathrm{CHO}$ cells can realize proper
A
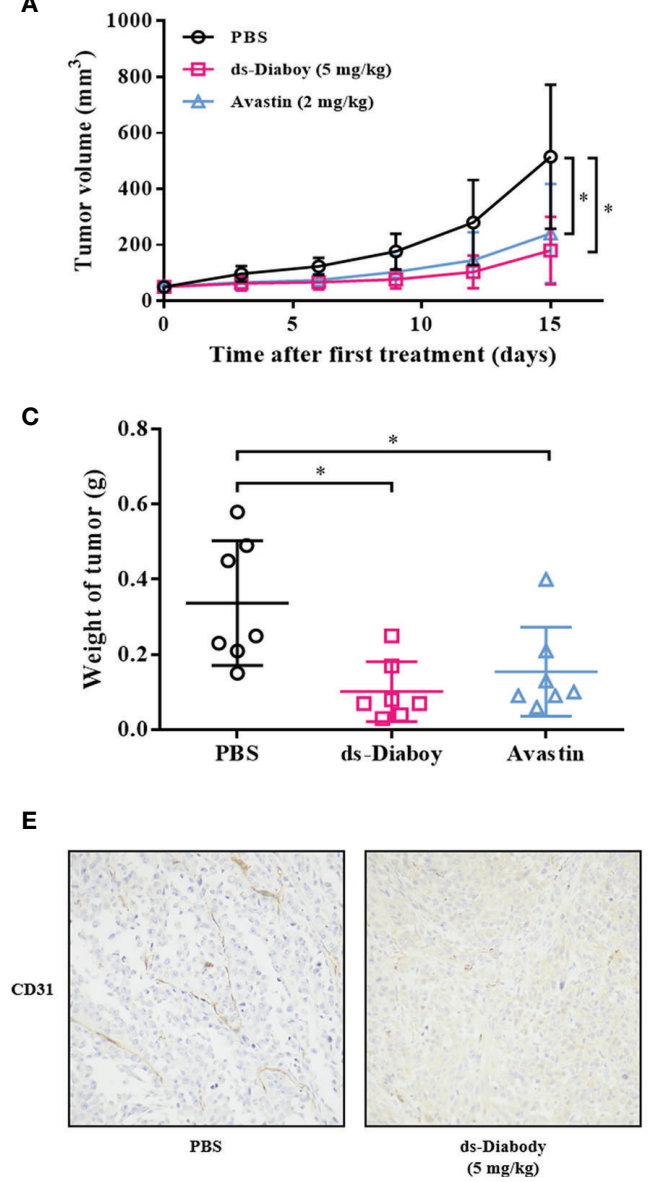

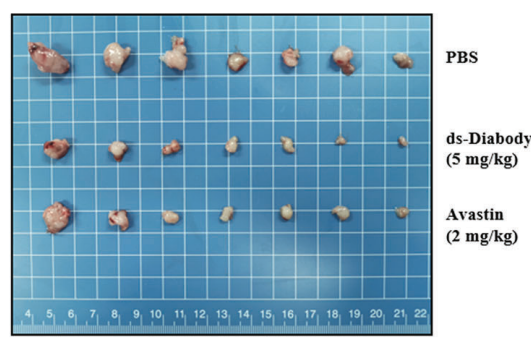

D

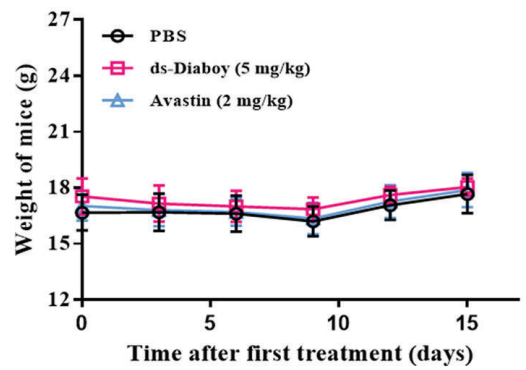

FIGURE 4 | The tumor inhibition of melanoma by ds-Diabody against FGF-2 in mice. The melanoma (A375) cells $\left(2.0 \times 10^{6}\right.$ cells in $100 \mu$ medium) were subcutaneously injected on the shoulder of BALB/c nude mice $(n=7)$. When the tumors were palpable, the ds-Diabody against FGF-2 were i.v. injected $(5 \mathrm{mg} / \mathrm{kg}$ in $100 \mu$ PBS) six times at 3 days intervals. (A) The tumor growth curve in nude mice in different groups. (B) The stripped tumors from nude mice in different groups. (C) The scatter diagram of tumor weights in different groups. (D) The weight curves of nude mice in different groups. (E) Immunohistochemistry analysis of microvessels of tumor tissues. The results presented as the mean $\pm \mathrm{SD}$ in triplicate. ${ }^{*} \mathrm{p}<0.05$ versus PBS Group. 
A

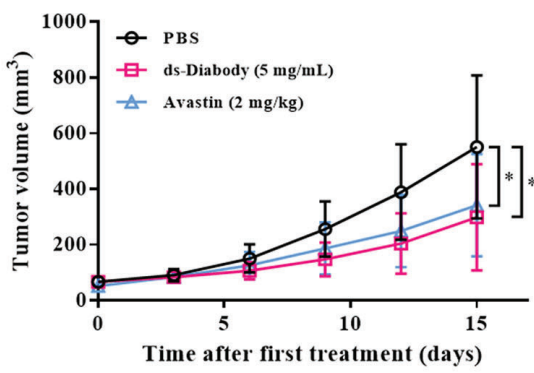

C

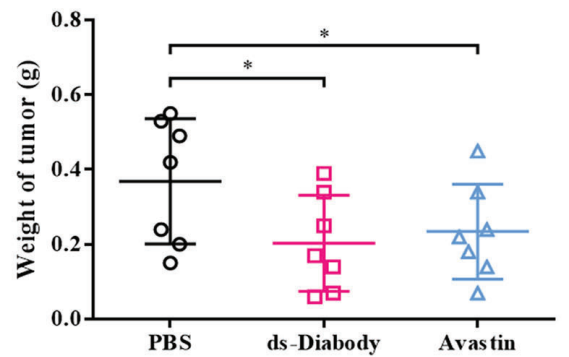

E

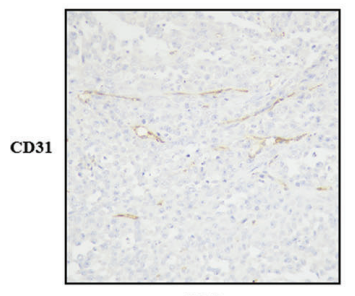

PBS

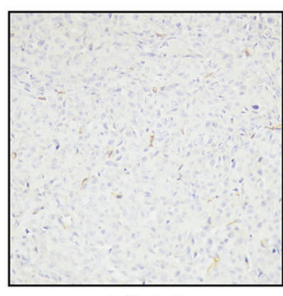

ds-Diabody

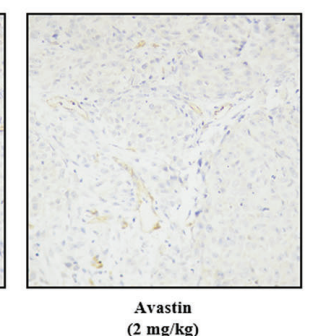

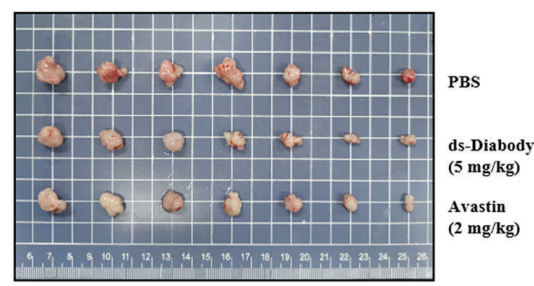

D

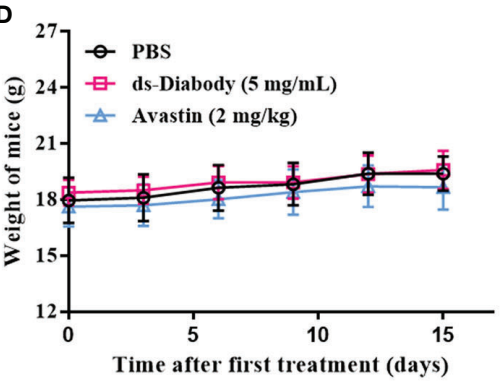

FIGURE 5 | The tumor inhibition of glioma by ds-Diabody against FGF-2 in mice. The glioma (U87) cells $\left(5.0 \times 10^{6}\right.$ cells in $100 \mu$ medium) were subcutaneously injected on the shoulder of BALB/c nude mice $(n=7)$. When the tumors were palpable, the ds-Diabody against FGF-2 were i.v. injected (5 mg/kg in $100 \mu L$ PBS) six times at 3 days intervals. (A) The tumor growth curve in nude mice in different groups. (B) The stripped tumors from nude mice in different groups. (C) The scatter diagram of tumor weights in different groups. (D) The weight curves of nude mice in different groups. (E) Immunohistochemistry analysis of microvessels of tumor tissues. The results presented as the mean \pm SD in triplicate. ${ }^{*} p<0.05$ versus PBS Group.

protein folding, posttranslational modifications, and glycosylation of recombinant proteins, but the expensiveness, long term culture and potential contamination with some viruses limited their wide use (26). Although the Pichia pastoris expression system is impressive and easy to use with well-defined process protocol, some degree of process optimization is required to achieve maximum production of the target protein.

The optimization in induction phase using substrate mixtures is crucial for improving the productivity of heterologous proteins with Pichia pastoris. Among the co-feeding strategies, co-carbon source (sorbitol) feeding strategy is as important as methanol feeding strategy. Sorbitol does not induce or repress AOX promoters; hence using sorbitol instead of glycerol co-fed with methanol could increase productivity and reduce induction time (18). In addition, the formation of toxic formaldehyde was reduced, and the cellular viability was enhanced by sorbitol/methanol co-feeding (27). Several studies revealed that methanol/sorbitol co-feeding increased the expression of the recombinant proteins (28-31). Consistent with this, using sorbitol in mixed substrate methods in our study, the induction time was reduced, and the production of ds-Diabody against FGF-2 was increased by 4.4 times.

FGF-2/FGFR system contributes to cancer progression by autocrine/paracrine stimulation of tumor cell proliferation and angiogenesis $(32,33)$. Elevated concentrations of FGF-2 and increased tumor microvascular density are potential markers of worse overall prognosis and abbreviated progression-free survival (19). FGF-2 was overexpressed in several tumors including melanoma and glioma (19-21). In this study, our data showed that ds-Diabody against FGF-2 could effectively inhibit melanoma and glioma in vivo. Li et al. (2018) suggested that LncRNA MEG3 can produce an almost 50\% decrease in A375 tumor growth through blocking Wnt signaling pathway (34). Zhou et al. found that GHRH antagonists produced an almost 60\% decrease in U87 tumor growth by multiple mechanisms including decreasing the release of FGF (35). Our data showed that the growth inhibition rate of A375 tumor and U87 tumor was about 70 and 45\%, respectively, and the angiogenesis inhibition rate of A375 tumor and U87 tumor was about 64 and 51\%, respectively. The inhibition 
effects of tumor growth and angiogenesis showed more effective compared to the Avastin group, which suggested that ds-Diabody was a potential anti-tumor drug for tumor therapy.

To identify the inhibition mechanism of ds-Diabody on the growth of A375 and U87 cells, the potential signal transduction pathways were detected. It was shown that the ds-Diabody against FGF-2 could effectively inhibit the proliferation, migration, and invasion of the A375 and U87 cells and block the phosphorylation activation of Akt and MAPK in a dose-dependent manner. MAPK/ ERK and PI3K/Akt are important signaling pathways in a variety of cellular processes, including cell proliferation and migration in tumor cells (36). Currently, several available inhibitors against the components of MAPK/ERK pathway have led to unprecedented clinical benefits in combating metastatic melanoma and glioma (37). Previous study showed that drugs targeting the MAPK/ERK pathway have profoundly changed the landscape of melanoma therapy (38). Similarly, several studies reported that MAPK/ERK signaling pathway involves in the migration and invasion of glioma cells (37). In our study, we found that MAPK/ERK and PI3K/AKt signals were significantly inhibited by the ds-Diabody. Therefore, our study discovered a possible upstream event through which dsDiabody exerted its anti-melanoma and glioma activity, although the precise mechanism needs further investigation.

Pathological angiogenesis plays a critical role in cancer and the anti-angiogenesis treatment has been also considered as a systemic strategy for cancer therapy (39). FGF-2 is an important tumor angiogenesis factor, and targeting FGF-2 can inhibit tumor angiogenesis and tumor growth. In our study, it was shown that the expression of CD31, a marker of vascularity, was decreased obviously in tumor tissues treated by ds-Diabody against FGF-2. The ds-Diabody against FGF-2 might possess significant effect on tumor therapy, compared to Avastin, and could be considered as a potential candidate for cancer therapy.

To summarize, the present study reported a fully human dsDiabody against FGF-2 expressed in Pichia pastoris, which possessed high antigen binding ability and anti-tumor activity in vitro and in vivo through inhibiting angiogenesis. Further studies will be necessary to study the ds-Diabody mechanisms and compare the efficacy with Avastin in other xenograft models. Based on its high efficiency and low toxicity, ds-Diabody against FGF-2 may provide an effective strategy for cancer therapy.

\section{CONCLUSION}

In conclusion, co-feeding of sorbital and methanol during the induction phase of Pichia pastoris fermentation was more

\section{REFERENCES}

1. Hafeez U, Parakh S, Gan HK, Scott AM. Antibody-Drug Conjugates for Cancer Therapy. Molecules (2020) 25(20):4764. doi: 10.3390/molecules25204764

2. Stantic M, Sakil HA, Zirath H, Fang T, Sanz G, Fernandez-Woodbridge A, et al. TAp73 suppresses tumor angiogenesis through repression of proangiogenic cytokines and HIF-1 $\alpha$ activity. Proc Natl Acad Sci (2015) 112(1):220-5. doi: 10.1073/pnas.1421697112 favorable than feeding of methanol and resulted in higher production of ds-Diabody against FGF-2. The ds-Diabody against FGF-2 inhibited the proliferation, migration, and invasion of A375 cells and U87 cells by decreasing the MAPK/ Akt signal pathways. Additionally, in xenograft models, dsDiabody against FGF-2 effectively suppressed melanoma and glioma tumo growth and angiogenesis. The ds-Diabody targeting FGF-2 may provide an effective strategy for cancer therapy.

\section{DATA AVAILABILITY STATEMENT}

The raw data supporting the conclusions of this article will be made available by the authors, without undue reservation.

\section{ETHICS STATEMENT}

The animal study was reviewed and approved by Institutional Animals Care and Use Committee on animal research in Jinan University, Guangzhou, China.

\section{AUTHOR CONTRIBUTIONS}

$\mathrm{SZ}, \mathrm{JH}$, and $\mathrm{LZ}$ designed and performed the experiments and drafted the manuscript. JG participated in the experiments. QS, YC, JCZ, HZ, YD, and WZ were involved in resource collection. ND and JFZ supervised this study. All authors contributed to the article and approved the submitted version.

\section{FUNDING}

This research was supported by grants from The Natural Science Foundation of China (81972705), Science and Technology Planning Project of Guangdong Province (2015B020211009, 2016A010105008) and Science and Technology Planning Project of Guangzhou City (201604020099).

\section{SUPPLEMENTARY MATERIAL}

The Supplementary Material for this article can be found online at: https://www.frontiersin.org/articles/10.3389/fonc.2021. 585457/full\#supplementary-material

3. Jiang X, Wang J, Deng X, Xiong F, Zhang S, Gong Z, et al. The role of microenvironment in tumor angiogenesis. J Exp Clin Cancer Res (2020) 39(1). doi: 10.1186/s13046-020-01709-5

4. Malapelle U, Rossi A. Emerging angiogenesis inhibitors for non-small cell lung cancer. Expert Opin Emerg Drugs (2019) 24(2):71-81. doi: 10.1080/ 14728214.2019.1619696

5. Folkman J. Angiogenesis: an organizing principle for drug discovery? Nat Rev Drug Discovery (2007) 6(4):273-86. doi: 10.1038/nrd2115 
6. Hui Q, Jin Z, Li X, Liu C, Wang X. FGF Family: From Drug Development to Clinical Application. Int J Mol Sci (2018) 19(7). doi: 10.3390/ijms19071875

7. Li Q, Gao S, Yu Y, Wang W, Chen X, Wang R, et al. A novel bFGF antagonist peptide inhibits breast cancer cell growth. Mol Med Rep (2012) 6(1):210-4. doi: $10.3892 / \mathrm{mmr} .2021 .882$

8. Su G, Meyer K, Nandini CD, Qiao D, Salamat S, Friedl A. Glypican-1 is frequently overexpressed in human gliomas and enhances FGF-2 signaling in glioma cells. Am J Pathol (2006) 168(6):2014-26. doi: 10.2353/ ajpath.2006.050800

9. Li X, Wang C, Xiao J, McKeehan WL, Wang F. Fibroblast growth factors, old kids on the new block. Semin Cell Dev Biol (2016) 53:155-67. doi: 10.1016/ j.semcdb.2015.12.014

10. Akl MR, Nagpal P, Ayoub NM, Tai B, Prabhu SA, Capac CM, et al. Molecular and clinical significance of fibroblast growth factor 2 (FGF2 /bFGF) in malignancies of solid and hematological cancers for personalized therapies. Oncotarget (2016) 7(28):44735-62. doi: 10.18632/oncotarget.8203

11. Presta M, Chiodelli P, Giacomini A, Rusnati M, Ronca R. Fibroblast growth factors (FGFs) in cancer: FGF traps as a new therapeutic approach. Pharmacol Ther (2017) 179:171-87. doi: 10.1016/j.pharmthera.2017.05.013

12. Navid S, Fan CO, Flores-Villanueva P, Generali D, Li Y. The Fibroblast Growth Factor Receptors in Breast Cancer: from Oncogenesis to Better Treatments. Int J Mol Sci (2020) 21(6). doi: 10.3390/ijms21062011

13. Katoh M, Nakagama H. FGF receptors: cancer biology and therapeutics. Med Res Rev (2014) 34(2):280-300. doi: 10.1002/med.21288

14. Fearon AE, Gould CR, Grose RP. FGFR signalling in women's cancers. Int $J$ Biochem Cell Biol (2013) 45(12):2832-42. doi: 10.1016/j.biocel.2013.09.017

15. Robinson ES, Khankin EV, Karumanchi SA, Humphreys BD. Hypertension induced by vascular endothelial growth factor signaling pathway inhibition: mechanisms and potential use as a biomarker. Semin Nephrol (2010) 30 (6):591-601. doi: 10.1016/j.semnephrol.2010.09.007

16. Sun HD, Malabunga M, Tonra JR, DiRenzo R, Carrick FE, Zheng H, et al. Monoclonal antibody antagonists of hypothalamic FGFR1 cause potent but reversible hypophagia and weight loss in rodents and monkeys. Am J Physiol Endocrinol Metab (2007) 292(3):E964-76. doi: 10.1152/ajpendo.00089.2006

17. Cai Y, Zhang J, Lao X, Jiang H, Yu Y, Deng Y, et al. Construction of a disulfide-stabilized diabody against fibroblast growth factor-2 and the inhibition activity in targeting breast cancer. Cancer Sci (2016) 107 (8):1141-50. doi: 10.1111/cas.12981

18. Orman MA, Calik P, Ozdamar TH. The influence of carbon sources on recombinant-human- growth-hormone production by Pichia pastoris is dependent on phenotype: a comparison of Muts and Mut+ strains. Biotechnol Appl Biochem (2009) 52(Pt 3):245-55. doi: 10.1042/ BA20080057

19. Go RS, Lee SJ, Shin D, Callister SM, Jobe DA, Conry RM, et al. ECOG Phase II Trial of Graded-Dose Peginterferon -2b in Patients with Metastatic Melanoma Overexpressing Basic Fibroblast Growth Factor (E2602). Clin Cancer Res (2013) 19(23):6597-604. doi: 10.1158/1078-0432.CCR-13-1414

20. Jimenez-Pascual A, Mitchell K, Siebzehnrubl FA. FGF2: a novel druggable target for glioblastoma? Expert Opin Ther Targets (2020) 24(4):311-8. doi: 10.1080/14728222.2020.1736558

21. Rezzola S, Ronca R, Loda A, Nawaz MI, Tobia C, Paganini G, et al. The Autocrine FGF/FGFR System in both Skin and Uveal Melanoma: FGF Trapping as a Possible Therapeutic Approach. Cancers (Basel) (2019) 11(9). doi: $10.3390 /$ cancers 11091305

22. Karbalaei M, Rezaee SA, Farsiani H. Pichia pastoris : A highly successful expression system for optimal synthesis of heterologous proteins. J Cell Physiol (2020) 235(9):5867-81. doi: 10.1002/jcp.29583

23. Tachioka M, Sugimoto N, Nakamura A, Sunagawa N, Ishida T, Uchiyama T, et al. Development of simple random mutagenesis protocol for the protein expression system in Pichia pastoris. Biotechnol Biofuels (2016) 9:199. doi: 10.1186/s13068-016-0613-z

24. Ma Y, Lee C, Park J. Strategies for Optimizing the Production of Proteins and Peptides with Multiple Disulfide Bonds. Antibiotics (2020) 9(9):541. doi: 10.3390/antibiotics9090541
25. Nylen A, Chen MT. Production of Full-Length Antibody by Pichia pastoris. Methods Mol Biol (2018) 1674:37-48. doi: 10.1007/978-1-4939-7312-5_3

26. Ha S, Wang Y, Rustandi RR. Biochemical and biophysical characterization of humanized IgG1 produced inPichia pastoris. mAbs (2014) 3(5):453-60. doi: 10.4161/mabs.3.5.16891

27. Wang B, Nesbeth D, Keshavarz-Moore E. Sorbitol/methanol mixed induction reduces process impurities and improves centrifugal dewatering in Pichia pastoris culture. Enzyme Microb Technol (2019) 130:109366. doi: 10.1016/ j.enzmictec.2019.109366

28. Gao M, Li Z, Yu RS, Wu JR, Zheng ZY, Shi ZP, et al. Methanol/sorbitol cofeeding induction enhanced porcine interferon- $\alpha$ production by P. Pastoris Associated Energy Metab Shift Bioprocess Biosyst Eng (2012) 35(7):1125-36. doi: 10.1007/s00449-012-0697-1

29. Wang Z, Wang Y, Zhang D, Li J, Hua Z, Du G, et al. Enhancement of cell viability and alkaline polygalacturonate lyase production by sorbitol cofeeding with methanol in Pichia pastoris fermentation. Bioresour Technol (2010) 101(4):1318-23. doi: 10.1016/j.biortech.2009.09.025

30. Zhu T, You L, Gong F, Xie M, Xue Y, Li Y, et al. Combinatorial strategy of sorbitol feeding and low-temperature induction leads to high-level production of alkaline beta-mannanase in Pichia pastoris. Enzyme Microb Technol (2011) 49(4):407-12. doi: 10.1016/j.enzmictec.2011.06.022

31. Celik E, Calik P, Oliver SG. Fed-batch methanol feeding strategy for recombinant protein production by Pichia pastoris in the presence of cosubstrate sorbitol. Yeast (2009) 26(9):473-84. doi: 10.1002/yea.1679

32. Wang Y, Becker D. Antisense targeting of basic fibroblast growth factor and fibroblast growth factor receptor-1 in human melanomas blocks intratumoral angiogenesis and tumor growth. Nat Med (1997) 3(8):887-93. doi: 10.1038/ nm0897-887

33. Zhong J, Zhang S, Zhang L, Cai Y, Deng Y, Zheng Q, et al. Fine epitope mapping of a human disulphide-stabilized diabody against fibroblast growth factor-2. J Biochem (2019) 165(6):487-95. doi: 10.1093/jb/mvy122

34. Li P, Gao Y, Li J, Zhou Y, Yuan J, Guan H, et al. LncRNA MEG3 repressed malignant melanoma progression via inactivating Wnt signaling pathway. $J$ Cell Biochem (2018) 119(9):7498-505. doi: 10.1002/jcb.27061

35. Jaszberenyi M, Schally AV, Block NL, Zarandi M, Cai RZ, Vidaurre I, et al. Suppression of the proliferation of human U-87 MG glioblastoma cells by new antagonists of growth hormone-releasing hormone in vivo and in vitro. Target Oncol (2013) 8(4):281-90. doi: 10.1007/s11523-013-0264-y

36. Zhou H, Li XM, Meinkoth J, Pittman RN. Akt regulates cell survival and apoptosis at a postmitochondrial level. J Cell Biol (2000) 151(3):483-94. doi: $10.1083 /$ jcb.151.3.483

37. Li B, Wang F, Liu N, Shen W, Huang T. Astragaloside IV inhibits progression of glioma via blocking MAPK/ERK signaling pathway. Biochem Biophys Res Commun (2017) 491(1):98-103. doi: 10.1016/j.bbrc.2017.07.052

38. Marchetti P, Trinh A, Khamari R, Kluza J. Melanoma metabolism contributes to the cellular responses to MAPK/ERK pathway inhibitors. Biochim Biophys Acta (BBA) - Gen Subj (2018) 1862(4):999-1005. doi: 10.1016/ j.bbagen.2018.01.018

39. Datta M, Via LE, Kamoun WS, Liu C, Chen W, Seano G, et al. Anti-vascular endothelial growth factor treatment normalizes tuberculosis granuloma vasculature and improves small molecule delivery. Proc Natl Acad Sci U.S.A. (2015) 112(6):1827-32. doi: 10.1073/pnas.1424563112

Conflict of Interest: The authors declare that the research was conducted in the absence of any commercial or financial relationships that could be construed as a potential conflict of interest.

Copyright $\odot 2021$ Zhang, Huang, Zhang, Gu, Song, Cai, Zhong, Zhong, Deng, Zhu, Zhao and Deng. This is an open-access article distributed under the terms of the Creative Commons Attribution License (CC BY). The use, distribution or reproduction in other forums is permitted, provided the original author(s) and the copyright owner(s) are credited and that the original publication in this journal is cited, in accordance with accepted academic practice. No use, distribution or reproduction is permitted which does not comply with these terms. 